\title{
Postulantes e ingresantes a la Facultad de Medicina de la Universidad Peruana Cayetano Heredia en el periodo 1962- 1994.
}

\section{Applicants and students entering the School of Medicine of the Universidad Peruana Cayetano Heredia.}

\author{
Risco de Dominguez Graciela ${ }^{1}$. \\ ${ }^{1}$ Profesora Principal del Departamento de Ciencias Fisiológicas de la Universidad \\ Peruana Cayetano Heredia. Lima, Perú.
}

\section{RESUMEN}

Se analiza la evolución de las características socioeconómicas y los antecedentes educacionales de postulantes e ingresantes a la Facultad de Medicina en la Universidad Peruana Cayetano Heredia (UPCH) entre 1962 y 1994. También se establecen comparaciones con información similar de otras universidades del país, encontrando similitudes en la evolución del número de postulantes. En la UPCH las vacantes de medicina se ha mantenido cerca de 70 desde 1962 hasta 1991, período en el cual las necesidades de médicos se incrementaron y la competencia por una vacante de medicina llegó a ser 60:1, muy superior al promedio de las otras universidades. La mayoría de los postulantes a la UPCH solicitan admisión a medicina y provienen de Lima y Callao. El estudio muestra que entre los postulantes a medicina se han incrementado los jóvenes de 15 a 16 años, las mujeres, los egresados de colegios particulares y los hijos de padres con educación superior. Los ingresantes muestran características similares, excepto que la edad es mayor de 18 años y que el intervalo secundaria universidad y el número de postulaciones hasta lograr el ingreso se ha incrementado. Se proponen razones que explican los cambios observados. (Rev Med Hered 1995; 6: 59-71).

PALABRAS CLAVE: Admisión, postulantes, ingresantes, características socioeconómicas, antecedentes educacionales.

\section{SUMMARY}

A study is made on the socio-economic characteristic and educational background of the applicants and students entering the School of Medicine of the Universidad Peruana Cayetano Heredia (UPCH); the study covered the period from 1962 to 1994, and it also compared related information from other universities in our country, showing similar variations in the numbers of applicants. In the UPCH the openings for medicine remained nearly 70 since 1962 until 1991, period in which the need for physicians increased, and the competition for entering reached a 60:1 proportion, stiffer than in any other university of the country. Most of the applicants to UPCH seek admittance to medical school and come from the metropolitan area of Lima. The study also shows an 
increasing number of younger applicants (15-16 years), of women, and of private schools graduates, whose parents have higher education. Entering students show similar characteristics, except that their age is higher than 18 years and that the interval between the end of school and the admittance to the university is increasing. A discussion is made of the observed findings. (Rev Med Hered 1995; 6: 59-71).

KEY WORDS: Admission, applicants, entering students, socioeconomic characteristics, educational background.

\section{INTRODUCCIÓN}

La UPCH, fundada en 1961, desde el inicio dio gran importancia a la adecuada selección de alumnos, en base a sus conocimientos y aptitudes académicas, por considerarla un requisito indispensable para el logro de la excelencia académica. La primera Comisión Técnica de Selección tuvo entre sus miembros a un grupo de docentes universitarios con varios años de experiencia en el perfeccionamiento de los métodos de selección de alumnos para ingreso a la carrera de medicina (1). La experiencia del grupo permitió establecer, desde el inicio, una metodología de trabajo que ha conducido al continuo perfeccionamiento del proceso de selección, en base a los resultados de la investigación sobre el mismo.

En 1985, ante el convencimiento de que para lograr estudiantes de alto nivel académico era insuficiente actuar únicamente a nivel del perfeccionamiento del proceso de selección, se creó en la UPCH el Centro de Estudios Pre Universitarios (CEPU). El notorio y progresivo deterioro de la educación escolar en los 25 años transcurridos desde la fundación de la Universidad, hacía indispensable intervenir también a nivel de la preparación del postulante. A partir de 1987 se asigna el 25\% de las vacantes de ingreso a la Universidad a los mejores alumnos del ciclo anual del CEPU, modalidad llamada del Ingreso Directo, la cual coexistió con el Examen de mejor rendimiento académico en la UPCH de los alumnos provenientes del Ingreso Directo, fue la principal razón por la que, a partir de 1991, acordó que todas las vacantes de ingreso a la Universidad se asignarían mediante Ingreso Directo, después de un ciclo semestral de estudios en el CEPU, que se ofrecería dos veces al año. Hemos llamado Ingreso Directo Exclusivo a esta modalidad de admisión.

Durante los 33 años que abarca el estudio la población del Perú se ha incrementado de 10 millones a 22 millones (2), y el país ha experimentado profundos cambios políticos económicos y sociales, que han afectado seriamente a la población, en especial a la juventud.

En el campo de la educación superior, la matrícula universitaria se expandió, de 30,000 alumnos en 1960, a 374,000 en 1993 (3,4). Si se relacionan estas cifras con el incremento de la población de 15 a 24 años de edad se obtiene la Tasa de Escolarización Superior, la cual se incrementó de $2.3 \%$ en 1961 a $20.4 \%$ en $1993(2,4)$, llegando a niveles de masificación. La matrícula en universidades privadas que representaba el $10 \%$ en 1961 a $20.4 \%$ en $1993(2,4)$, llegando a niveles de masificación. La matrícula en universidades privadas que representaba el $10 \%$ en 1961 ha pasado a representar el $37 \%$ en $1993(2,4)$. El número de universidades ha aumentado de 8 públicas y 1 privada en 1960, a 28 públicas y 27 privadas en $1994(2,4)$. El nivel de educación superior se ha 
expandido notablemente además por el incremento de número de Institutos Superiores Tecnológicos, más marcado a partir de 1983 (5), los cuales captan a un importante sector de los egresados de secundaria.

Cambios similares a los descritos se han producido en la educación superior en toda América Latina a partir de 1950, como lo describe Brunner (6): "Si hay algo que llama la atención en la evolución reciente de la educación superior en América Latina es la explosión de los números: de establecimientos, de alumnos y de docentes".

La UPCH, que es parte de esta realidad, también sufrió el impacto de los cambios ocurridos, por ello presentamos este estudio cuyo objetivo es hacer un análisis comparativo de las características socioeconómicas y los antecedentes educacionales de los postulantes e ingresantes a la Facultad de Medicina de la Universidad Peruana Cayetano Heredia (UPCH), desde 1962 hasta 1994. También analizaremos la evolución en el número de postulantes y de vacantes de medicina en el período del estudio.

Este estudio permitirá tener una visión de la evolución de la realidad socioeconómica y educativa de los jóvenes egresados de la educación secundaria que aspiran a realizar estudios en nuestra universidad. Dado que son pocos los estudios de este tipo en nuestro medio, creemos que la información aquí presentada será también de interés para otras universidades y facultades de medicina del país.

\section{MATERIALES Y MÉTODOS}

Para realizar este estudio se contó con la siguiente información sobre los postulantes e ingresantes a la UPCH:

- Informe sobre los procesos de selección 1962 y 1964, elaborado por la primera Comisión Técnica de Selección de la UPCH (1). El informe fue elaborado en base a una muestra aleatoria, estadísticamente representativa del total de postulantes. Para 1962 la muestra constó de 106 postulantes, de un total de 529, para 1963 se tomaron 95 de 479 postulantes, y para 1964 fueron 112 de 559 postulantes. Los datos biográficos se obtuvieron de la ficha llenada por los antecedentes educacionales se extrajeron de los certificados de educación secundaria

- Informes elaborados por las respectivas comisiones de ingreso, sobre la Encuesta Socioeconómica y Demográfica, aplicada a los postulantes en los años 1981, 1982 y 1983 (7). La Encuesta fue aplicada a los postulantes al momento de la inscripción. Para la respuesta se utilizaron tarjetas ópticas que fueron leídas y procesadas por computadora. Estos informes se elaboraron sobre la base de la población total de postulantes.

- Informe: “Análisis Comparativo de las Características Socioeconómicas de los Postulantes e Ingresantes a la UPCH a lo largo de tres años: 1981-1983” (8).

- Informes estadísticos elaborados por las respectivas comisiones de ingreso, sobre la Encuesta Socioeconómica aplicada a los postulantes durante los años 1990 a 1993. El contenido de la encuesta y su procesamiento fueron similares a los del período de 1981-83. Los informes comprenden al total de la población para cada año. A partir 
de 1992, al ser el proceso de admisión semestral, la aplicación de la encuesta también lo fue pero para efectos de este trabajo hemos integrado los datos de dos semestres en un dato anual único.

- Listados de Orden de Mérito publicados por las respectivas comisiones de ingreso entre 1979 y 1994.

- Expediente de los alumnos de la Facultad de Medicina para los años 1989 a 1991-I. Expedientes de los alumnos del Ciclo Básico para los años 1991II a 1993. De ellos hemos tomado los datos biográficos y los antecedentes educacionales de los ingresantes entre 1989 y 1993.

En base a esta información se realizó un estudio descriptivo comparativo de las características de los postulantes e ingresantes a la UPCH, tomando en cuenta las siguientes variables: Número de postulantes/ingresantes, características socioeconómicas y antecedentes educacionales: sexo, edad, tipo y ubicación del colegio de procedencia, intervalo secundaria universidad, número de veces que postularon hasta alcanzar el ingreso de instrucción de los padres.

La información se presenta en forma de frecuencia absolutas y porcentajes. Para las variables socioeconómicas y los antecedes educacionales se calcula:

Porcentaje de Éxito al Ingreso $=\left(\mathrm{N}^{\mathrm{o}}\right.$ de ingresantes $/ \mathrm{N}^{\mathrm{o}}$ de postulantes $) \times 100$.

El Porcentaje de Éxito al Ingreso (P.E.I) indica el porcentaje de ingresantes de entre los postulantes que comparten cierta característica.

\section{RESULTADOS}

\section{Número de Postulantes}

En el cuadro $\mathrm{N}^{\mathrm{o}} 1$, se presenta el número y porcentaje de postulantes e ingresantes y la relación postulantes/ingresantes para las tres modalidades de admisión, tanto para la UPCH en total como para la Facultad de Medicina. La información presentada corresponde a los períodos 1962-64 y 1979-1994. 
POSTULANTES,INGRESANTES Y RELACION POSTULANTES/INGRESANTE

UPCH Y FACULTAD DE MEDICINA

\begin{tabular}{|c|c|c|c|c|c|c|c|c|}
\hline \multirow{3}{*}{ AÑo } & \multicolumn{5}{|c|}{ FACULTAD DE MEDICINA } & \multicolumn{3}{|c|}{ TOTAL UPCH } \\
\hline & \multicolumn{2}{|c|}{ Postulantes } & \multicolumn{2}{|c|}{ Ingresantes } & \multirow[t]{2}{*}{ Post/lngr. } & \multirow[t]{2}{*}{ Postulan. } & \multirow[t]{2}{*}{ Ingresan. } & \multirow[t]{2}{*}{ Post/lngr } \\
\hline & $\mathrm{n}$ & $\%$ & $\mathrm{n}$ & $\%$ & & & & \\
\hline \multicolumn{9}{|c|}{ EXAMEN DE ADMISION 1962-1994-I } \\
\hline 1962 & 529 & 100 & 70 & 100 & 8 & 529 & 70 & 8 \\
\hline 1963 & 479 & 100 & 60 & 100 & 8 & 479 & 60 & 8 \\
\hline 1964 & 559 & 100 & 60 & 100 & 9 & 559 & 60 & 9 \\
\hline : & : & : & : & : & : & : & : & : \\
\hline 1979 & 1590 & 84 & 56 & 44 & 28 & 1902 & 126 & 15 \\
\hline 1980 & 2388 & 79 & 61 & 48 & 39 & 3016 & 128 & 24 \\
\hline 1981 & 3190 & 80 & 69 & 50 & 46 & 4005 & 138 & 29 \\
\hline 1982 & 2877 & 80 & 71 & 54 & 41 & 3609 & 131 & 28 \\
\hline 1983 & 2781 & 81 & 73 & 51 & 38 & 3448 & 143 & 24 \\
\hline 1984 & 2569 & 85 & 73 & 50 & 35 & 3038 & 146 & 21 \\
\hline $1985(A)$ & 2350 & 87 & 67 & 47 & 35 & 2693 & 143 & 19 \\
\hline $1986(A)$ & 2786 & 89 & 50 & 47 & 56 & 3145 & 107 & 29 \\
\hline $1987(A)$ & 3224 & 84 & 50 & 45 & 64 & 3820 & 110 & 35 \\
\hline 1988 & 3164 & 78 & 51 & 50 & 62 & 4058 & 102 & 40 \\
\hline 1989 & 1641 & 75 & 46 & 34 & 36 & 2199 & 134 & 16 \\
\hline 1990 & 1970 & 76 & 59 & 40 & 33 & 2600 & 146 & 18 \\
\hline ا-1991 & 1794 & 73 & 60 & 43 & 30 & 2448 & 139 & 18 \\
\hline \multicolumn{9}{|c|}{ INGRESO DIRECTO 1987-1991-I } \\
\hline 1987 & 460 & 90 & 20 & 61 & 23 & 511 & 33 & 15 \\
\hline 1988 & 406 & 86 & 20 & 50 & 20 & 470 & 40 & 12 \\
\hline 1989 & 585 & 88 & 20 & 50 & 29 & 667 & 40 & 17 \\
\hline 1990 & 596 & 83 & 21 & 51 & 28 & 718 & 41 & 18 \\
\hline $1991-1$ & 742 & 83 & 22 & 41 & 34 & 894 & 54 & 17 \\
\hline & \multicolumn{8}{|c|}{ INGRESO DIRECTO EXCLUSIVO 1991-II A 1994-II } \\
\hline 1991-II & 726 & 78 & 30 & 40 & 24 & 928 & 75 & 12 \\
\hline 1992-I & 601 & 79 & 56 & 47 & 11 & 760 & 118 & 6 \\
\hline 1992-II & 677 & 66 & 85 & 46 & 8 & 1022 & 183 & 6 \\
\hline Total1992 & 1278 & 72 & 141 & 47 & 9 & 1782 & 301 & 6 \\
\hline I-1993 & 471 & 58 & 64 & 40 & 7 & 819 & 159 & 5 \\
\hline 1993-II & 603 & 65 & 78 & 48 & 8 & 933 & 162 & 6 \\
\hline Total 1993 & 1074 & 61 & 142 & 44 & 8 & 1752 & 321 & 5 \\
\hline I-1994 & 478 & 61 & 59 & 37 & 8 & 787 & 159 & 5 \\
\hline 1994-II & 634 & 70 & 88 & 47 & 7 & 911 & 189 & 5 \\
\hline Total1994 & 1112 & 65 & 147 & 42 & 8 & 1698 & 348 & 5 \\
\hline
\end{tabular}

Fuente: Listados de Comisión de Ingreso. (A)=Doble Opción Medicina-Ciencias

En 1962 postularon al Examen de Admisión 529 egresados de secundaria para ocupar las 70 vacantes que ofrecía la única facultad con que contaba la Universidad, la Facultad de Medicina. Entre 1963 y 1964 el número de postulantes mostró pocas variaciones en relación a 1962. En los años siguientes la Universidad incrementó su oferta de vacantes, con las de Bachillerato en Ciencias, a partir de 1967, y las de Estomatología a partir de 1972.

Entre 1979 y 1986 el número de postulantes a la UPCH varió entre 1900 y 4000, de ellos más de $80 \%$ postulaban a Medicina.

Entre 1987 y el primer semestre de 1991 a la modalidad de ingreso mediante el Examen de Admisión se añadió la modalidad de ingreso Directo, por la cual se otorgaron vacantes de ingreso a la UPCH a los mejores alumnos del Ciclo Anual del CEPU. En la Figura $\mathrm{N}^{\mathrm{o}} 1$, se muestra el total de postulantes por año para éste período el cual osciló entre un máximo de 4500 en 1988 y un mínimo de 2866 en 1989. 


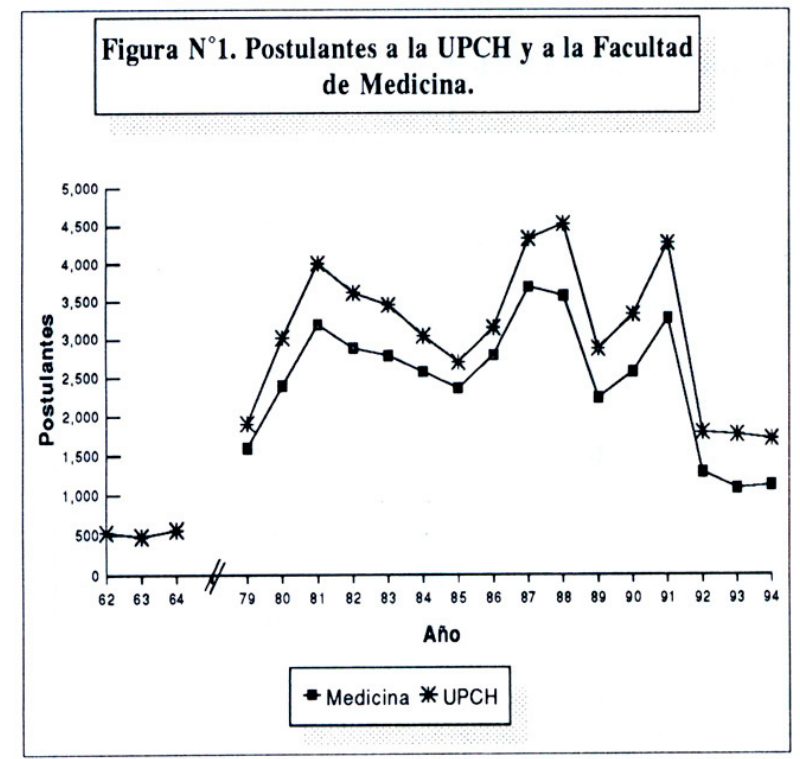

En 1991-II se inició la modalidad de Ingreso Directo Exclusivo, la cual se extendió hasta 1994. En éste período todos los postulantes debieron matricularse en un ciclo semestral de estudios en el 1800 por año, 60 a $70 \%$ de los cuales postularon a Medicina.

\section{Número de Integrantes.}

El número de ingresantes es igual al de vacantes ya que en todos los años se cubrió el total de vacantes ofrecidas. En el cuadro $\mathrm{N}^{\circ} 1$, observamos que el número de vacantes de medicina fué de 70 en 1962 descendió a 60 en los dos años siguientes. En el período 1979-1989 su número varió, entre un mínimo de 50 en 1986 y un máximo de 73 en 1983 y 1984. Entre 1987 y 1989, el 25\% de las vacantes de medicina se asignó al Ingreso Directo. El número de vacantes de medicina se incrementó a 80 en 1990 y luego, a partir de 1992, con el cambio de modalidad de admisión, se llega a 140 vacantes anuales. En la figura $\mathrm{N}^{\circ} 2$, se presenta la suma total de ingresantes por año y la relación Postulantes/Ingresantes.

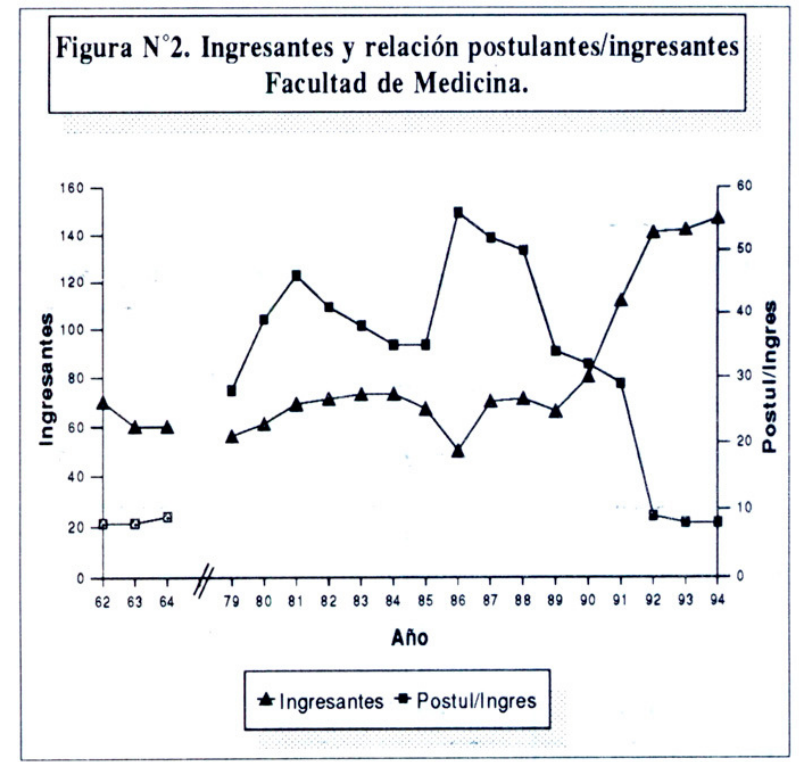




\section{Relación Postulantes/Ingresante}

En el cuadro $\mathrm{N}^{\mathrm{o}} 1$ y la figura $\mathrm{N}^{\mathrm{o}} 2$, observamos que en los años 1962-64 la relación Postulantes/Ingresantes fue de 8 , valor que se incrementó significativamente a lo largo de los años. Entre 1986 y 1988 esta relación alcanzó sus valores más altos: entre 56 y 64 postulantes por una vacantes de medicina en el Examen de Admisión. La competencia en el Ingreso Directo estuvo entre 20 y 34 postulantes por ingresante a medicina, y en el caso del Ingreso Directo Exclusivo, a partir de 1992, esta cifra volvió a los valores iniciales de 8 o 9 postulantes por ingresante. La relación Postulante/Ingresante para Medicina ha sido siempre superior a la de las otras dos facultades de la universidad.

\section{Características Socioeconómicas y Antecedentes Educacionales de los Postulantes e Ingresantes}

Para analizar la evolución de estas variables a lo largo del tiempo se cuenta con información de tres períodos: 1962-64, 1981-83 y 1990-93. A continuación se presenta la información sobre estas variables.

a) Sexo.- En el cuadro $\mathrm{N}^{0} 2$, se presenta el porcentaje de hombres y de mujeres entre los postulantes e ingresantes. Se observa un incremento en el porcentaje de mujeres a lo largo de los tres períodos estudiados, tanto entre postulantes como entre ingresantes. En el período 1962-64 el 17\% de los postulantes y el 13\% de los ingresantes fueron mujeres. En el período 1990-92 las mujeres constituyeron el 42\% de los postulantes y el 33\% de los ingresantes (Figura No3). El Porcentaje de Éxito al Ingreso (P.E.I.)

b) fue mayor par los hombres en los tres períodos estudiados.

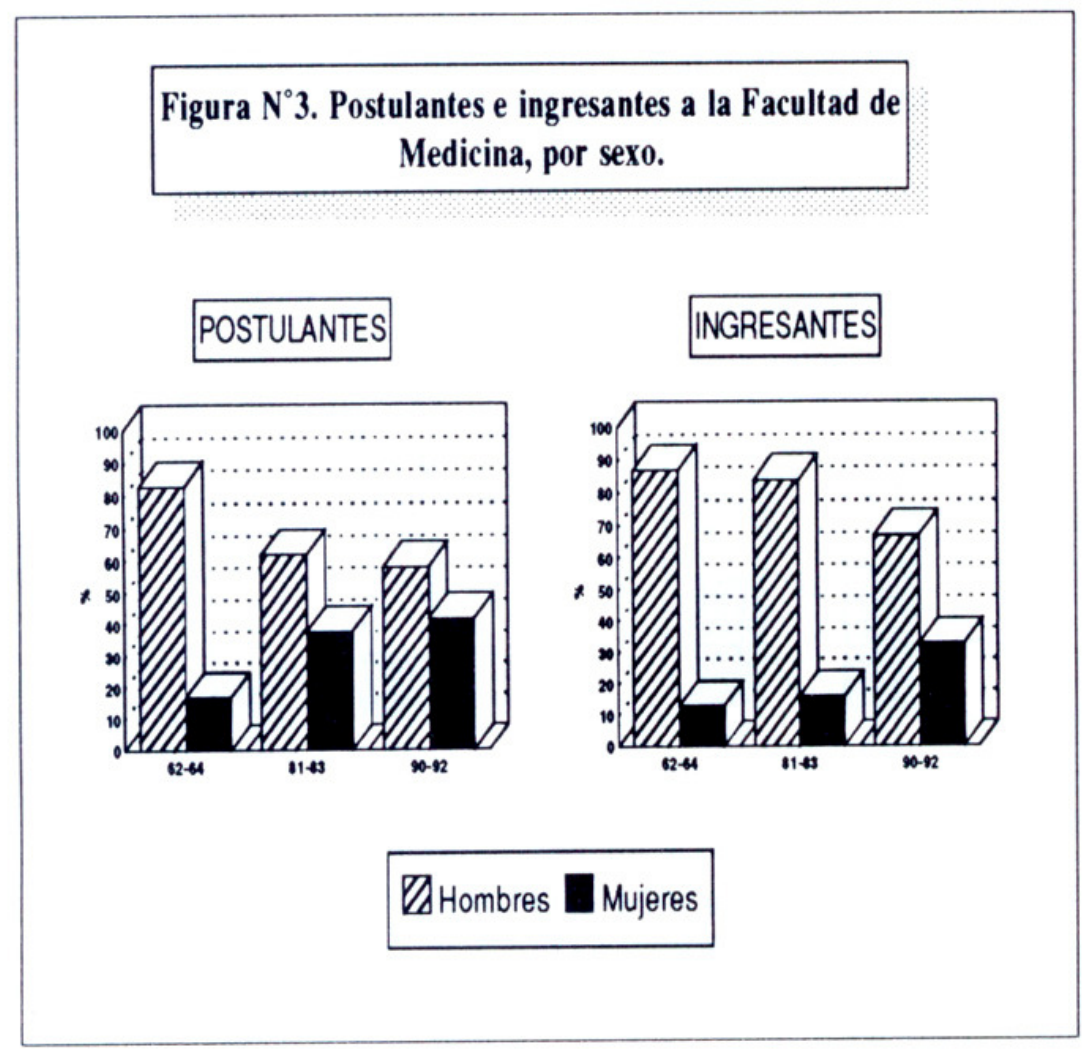


c) Edad.- Los datos se presentan en el cuadro $\mathrm{N}^{\mathrm{0}} 3$, en él observamos que entre los postulantes el porcentaje de jóvenes de 15 a 16 años de edad se ha incrementado de $11 \%$ en el período 1962-1964, a 29\% en el período 1990-93, a expensas de una reducción en el porcentaje de postulantes de 19 y más años. Entre los ingresantes (Figura $\mathrm{N}^{\mathrm{o}}$ 4), por el contrario, el porcentaje de jóvenes de 15-16 años ha disminuido de $32 \%$ en el período 1962-64, a 1\% entre 1990 y 1993, mientras que el porcentaje de ingresantes de 19 años o más se ha incrementado. Como consecuencia de lo anterior el porcentaje de ingresantes de 19 años o más, que era de $19 \%$ en el período 1962-64, el mayor P.E.I. lo tuvieron los postulantes de 15-16 años de edad, en los siguientes periodos observamos un desplazamiento de los valores altos del P.E.I. hacia los postulantes de mayor edad.

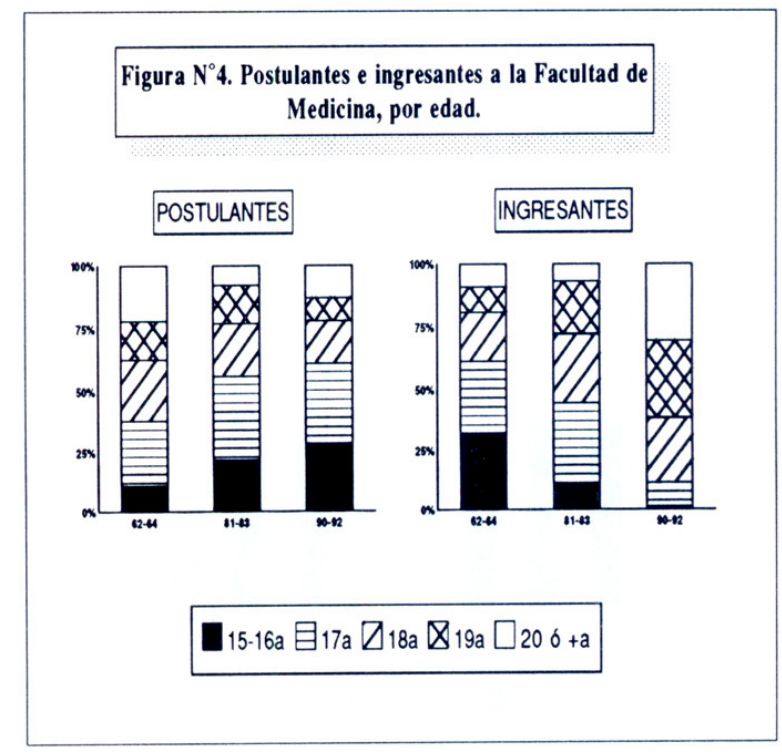

d) Tipo de Colegio de Procedencia.- (Cuadro No4 y Figura №5). En el 1962 el 50\% de los postulantes a la Facultad de Medicina terminó secundaria en un colegio estatal, porcentaje que ha ido disminuyendo con el tiempo hasta llegar al $27 \%$ en 1993. Entre los integrantes el porcentaje de egresados de colegios estatales disminuyó de $45 \%$ a $30 \%$ y a $15 \%$ e los tres períodos estudiados. El P.E.I. de los egresados de colegios estatales ha sido inferior al de los egresados de colegios particulares en los tres períodos. 


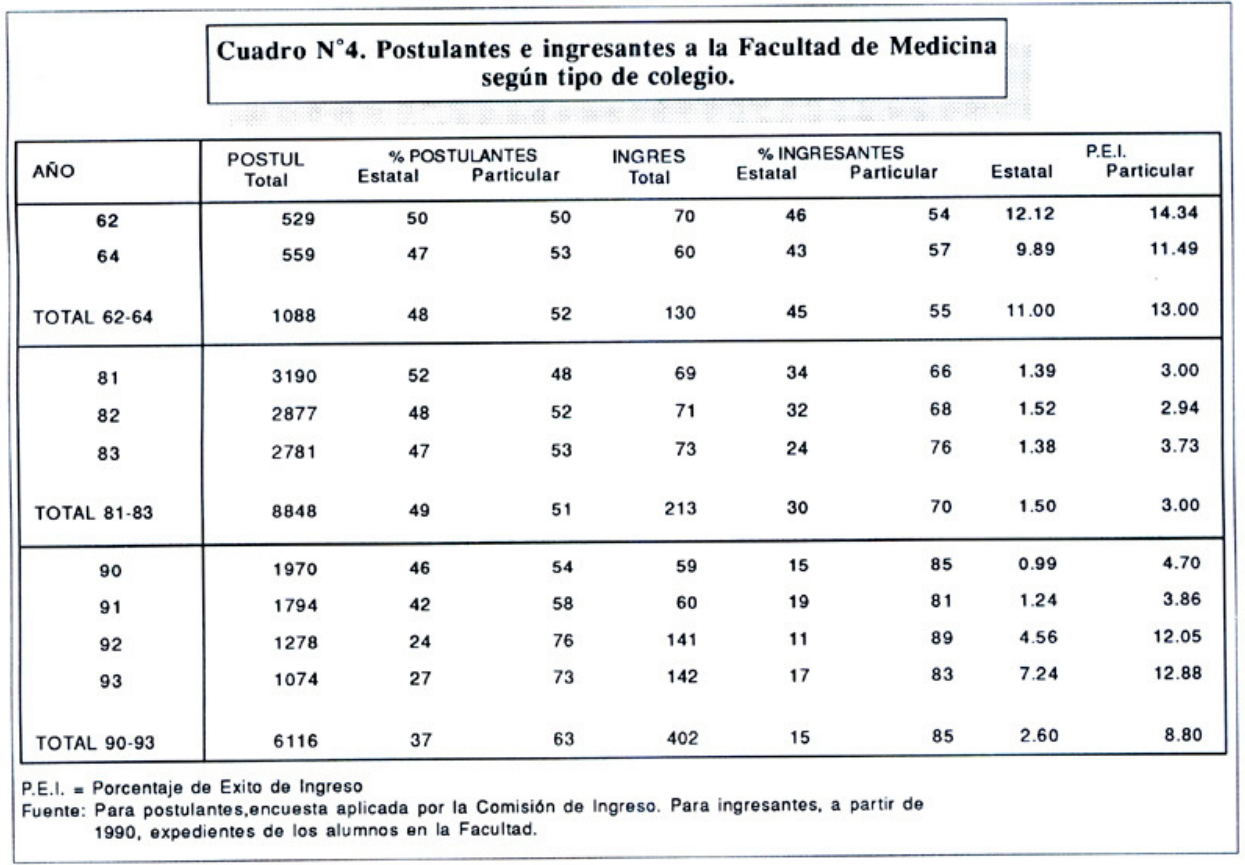

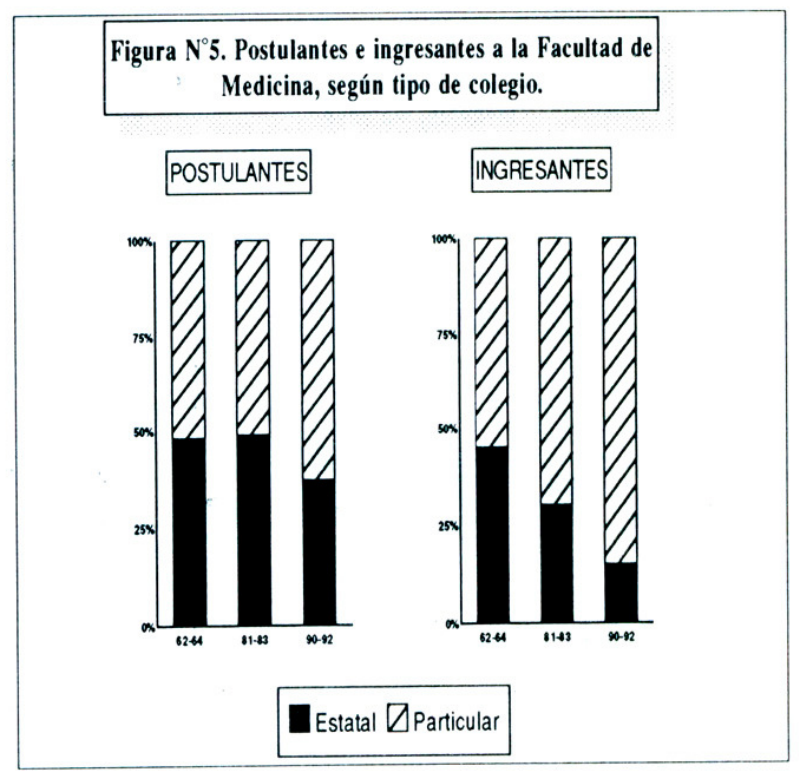

e) Ubicación del Colegio.- Como se observa en el cuadro №5, en los tres períodos, el $70 \%$ de los postulantes y el $80 \%$ de los ingresantes provino de colegios ubicados en Lima y Callao. 


\begin{tabular}{|c|c|c|c|c|}
\hline \multicolumn{5}{|c|}{$\begin{array}{l}\text { Cuadro } \mathrm{N}^{\circ} 5 \text {. Postulantes e ingresantes, según ubicación } \\
\text { del colegio de procedencia. Facultad de Medicina. }\end{array}$} \\
\hline \multirow[b]{2}{*}{ AÑo } & \multicolumn{2}{|c|}{$\%$ POSTULANTES } & \multicolumn{2}{|c|}{$\%$ INGRESANTES } \\
\hline & LIma-Callao & Otr Prov & Lima-Callao & Otr Prov \\
\hline 62 & 69 & 31 & 81 & 19 \\
\hline 64 & 71 & 29 & 77 & 23 \\
\hline 81 & 67 & 33 & 78 & 22 \\
\hline 82 & 66 & 34 & 77 & 23 \\
\hline 83 & 70 & 30 & 87 & 13 \\
\hline 90 & 58 & 42 & 84 & 16 \\
\hline 91 & 64 & 36 & 70 & 30 \\
\hline 92 & 71 & 29 & 84 & 16 \\
\hline
\end{tabular}

f) Intervalo Secundaria - Universidad.- Es el tiempo transcurrido entre el año en que el postulantes concluyó secundaria y el año en que postuló a la Universidad. Se cuenta con información para dos períodos: 1981-83 y 1990-93, como lo muestra el cuadro $\mathrm{N}^{\mathrm{o}} 6$ y la figura $\mathrm{N}^{\mathrm{o}} 6$. En los dos períodos se observa que entre los postulante predominan los que concluyeron secundaria un año antes de postular y entre los ingresantes los que concluyeron secundaria dos años antes. Se observa también que entre el primer y segundo período el porcentaje de ingresantes que concluyó secundaria el año anterior ha disminuido y se ha incrementado el porcentaje de los que concluyeron secundaria 4 ó más años antes de ingresar. El P.E.I. más bajo lo tienen los postulantes que terminaron secundaria un año antes de postular y el más alto los que terminaron secundaria 2 ó 3 años antes.

\begin{tabular}{|c|c|c|c|c|c|c|c|c|c|c|}
\hline \multicolumn{11}{|c|}{$\begin{array}{l}\text { Cuadro } \mathrm{N}^{\circ} 6 \text {. Postulantes e ingresantes a la Facultad de Medicina, } \\
\text { según intervalo secundaria-universidad. }\end{array}$} \\
\hline \multirow{2}{*}{ AÑO } & \multirow{2}{*}{$\begin{array}{l}\text { TOTAL } \\
\text { POSTUL }\end{array}$} & \multicolumn{3}{|c|}{ 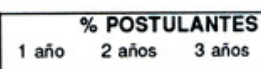 } & $40 \mathrm{mas}$ & $\begin{array}{c}\text { TOTAL } \\
\text { INGRES }\end{array}$ & \multicolumn{3}{|c|}{ 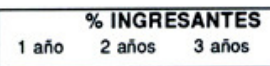 } & $46 \mathrm{mas}$ \\
\hline & & \begin{tabular}{|c|}
1 ando \\
50 \\
\end{tabular} & 28 & 12 & 10 & $\frac{1 \text { NGHES }}{69}$ & 20 & 44 & 28 & 8 \\
\hline 82 & 2877 & 52 & 25 & 11 & 12 & 71 & 26 & 40 & 21 & 13 \\
\hline 83 & 2781 & 49 & 28 & 11 & 12 & 73 & 21 & 50 & 20 & 9 \\
\hline TOTAL 81-83 & 8848 & 50 & 27 & 11 & 11 & 213 & 22 & 45 & 23 & 10 \\
\hline P.E.I. & & & & & & & 1 & 4 & 5 & 2 \\
\hline 90 & 1970 & 49 & 22 & 13 & 16 & 59 & 9 & 31 & 38 & 21 \\
\hline 91 & 1794 & 44 & 25 & 15 & 16 & 60 & 12 & 40 & 22 & 24 \\
\hline 93 & 1278 & 41 & 21 & 17 & 21 & 141 & 13 & 47 & 16 & 23 \\
\hline TOTAL $90-93$ & 5042 & 45 & 23 & 15 & 17 & 260 & 12 & 42 & 23 & 22 \\
\hline P.E.I. & & & & & & & 1.3 & 9 & 8 & 7 \\
\hline $\begin{array}{l}\text { P.E.I. }=\text { Porce } \\
\text { Fuente: Encu } \\
\text { de IC }\end{array}$ & $\begin{array}{l}\text { lica } \\
\text { nos }\end{array}$ & $F$ & $\begin{array}{l}\text { o. } \\
\text { nision } \\
\text { d. }\end{array}$ & Ingres & Para in & antes & rtir d & 990, ex & edientes & \\
\hline
\end{tabular}




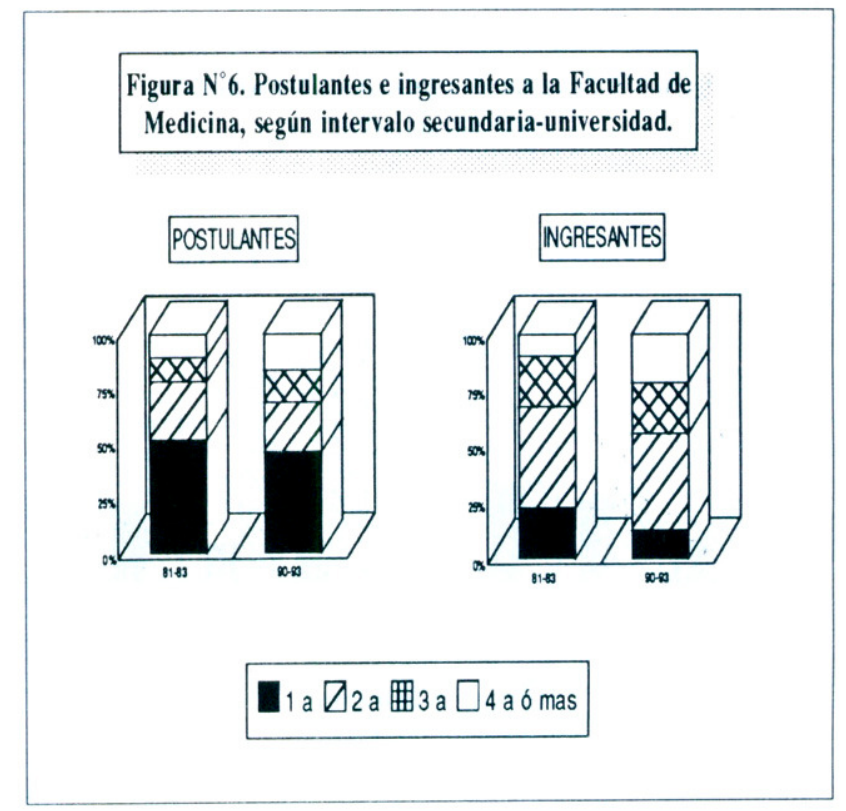

g) Número de Veces Que Postularon.- Contamos con información para los períodos 1981-83 y 1992-94 la cual se presenta en el cuadro $\mathrm{N}^{0} 7$ y la figura $\mathrm{N}^{\circ} 7$. En ambos períodos el mayor porcentaje de postulantes correspondió a los que postulaban por primera vez. Entre los ingresantes el porcentaje de los que postulaban por primera vez ha disminuido entre el primer y el segundo período, y se ha incrementado en de los que postulaban por tercera o cuarta vez. Debemos considerar que en período 1992-94 estuvo en vigencia la modalidad de Ingreso Directo Exclusivo, en la cual el ingreso a la UPCH fue semestral, a diferencia del período 1981-83 en que el cual el ingreso fue anual. Por lo tanto a igual número de postulaciones el tiempo transcurrido en el segundo período es la mitad que en el primer período. El mayor P.E.I. correspondió a los que postulaban por tercera o cuarta vez para los dos períodos. 


\begin{tabular}{|c|c|c|c|c|c|c|c|c|c|c|}
\hline \multirow[b]{3}{*}{ AÑO } & \multicolumn{8}{|c|}{$\begin{array}{c}\text { Cuadro } \mathrm{N}^{\circ} \text { 7. Postulantes e ingresantes a Medicina, } \\
\text { según número de veces que postularon. }\end{array}$} & \\
\hline & TOTAL & \multicolumn{4}{|c|}{$\%$ POSTULANTES } & \multirow{2}{*}{$\begin{array}{c}\text { TOTAL } \\
\text { INGRES }\end{array}$} & \multicolumn{2}{|c|}{$\%$ INGRESANTES } & & \\
\hline & POSTUL & $1 \mathrm{~V}$ & $2 \mathrm{~V}$ & $3 \mathrm{~V}$ & 4 y $5 \mathrm{~V}$ & & $1 \mathrm{~V}$ & $2 \mathrm{~V}$ & $3 \mathrm{~V}$ & 4 y $5 \mathrm{~V}$ \\
\hline 81 & 3190 & 74 & 19 & 6 & 1 & 69 & 32 & 44 & 20 & 4 \\
\hline 82 & 2877 & 71 & 22 & 6 & 1 & 71 & 33 & 45 & 20 & 2 \\
\hline 83 & 2781 & 68 & 23 & 7 & 2 & 73 & 25 & 56 & 15 & 4 \\
\hline TOTAL $81-83$ & 8848 & 71 & 21 & 6 & 1.6 & 213 & 30 & 48 & 19 & 3 \\
\hline P.E.I. & 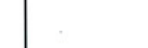 & & & & & & 1 & 5.5 & 7 & 5 \\
\hline 92 & 1278 & 33 & 27 & 27 & 10 & 141 & 15 & 33 & 52 & 0 \\
\hline 93 & 1074 & 66 & 21 & 10 & 3 & 142 & 17 & 32 & 23 & 28 \\
\hline 94 & 1112 & 58 & 21 & 15 & 6 & 147 & 24 & 42 & 22 & 11 \\
\hline TOTAL 92-94 & 3464 & 52 & 23 & 18 & 6.5 & 430 & 19 & 36 & 32 & 13 \\
\hline P.E.I. & & & & & & & 4.5 & 19 & 22 & 25 \\
\hline $\begin{array}{l}\text { P.E.I. }=\text { Porcentaje } \\
\text { Fuente: Encuesta } \\
\text { datos del }\end{array}$ & $\begin{array}{l}\text { e de exito de } \\
\text { aplicada po } \\
\text { CEPU. }\end{array}$ & eso & de & enes & a ino & es a & 1 & 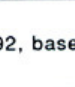 & & \\
\hline
\end{tabular}

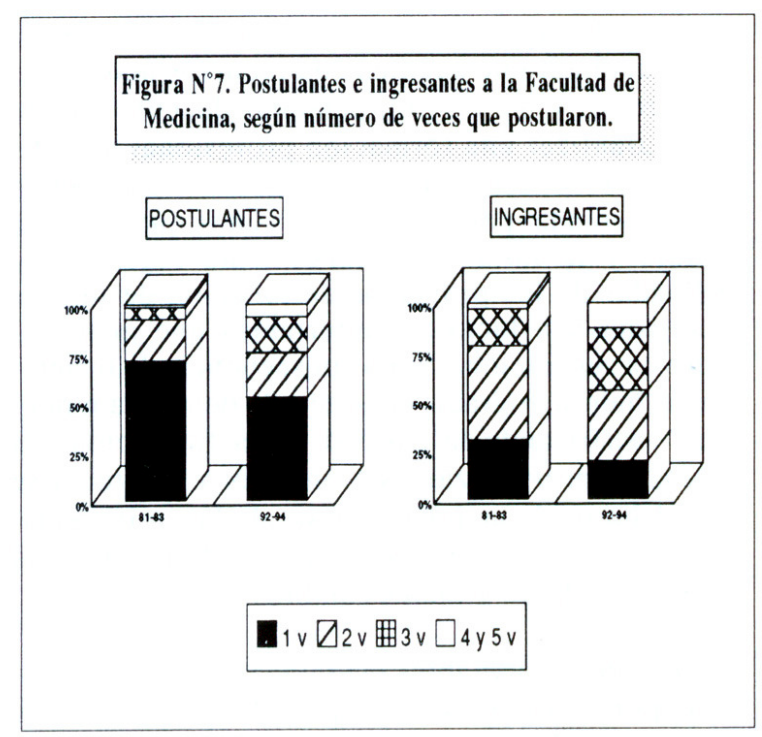

h) Grado de Instrucción de la Madre.- En el cuadro No8, observamos que entre los postulantes el porcentaje de madres con educación superior se ha incrementado, de $18 \%$ en el período 1962-64, a 45\% en el período 1990-93. Entre los ingresantes el porcentaje de madres con educación superior se ha incrementado en mayor proporción, de 23\% en 1962-64 a 54\% en el período 1990-93. Para cada período el P.E.I. fue mayor para el grupo de postulantes cuyas madres tenían educación superior.

i) Grado de Instrucción del Padres.- En el cuadro $N^{\circ} 8$, observamos que entre los postulantes el grado de instrucción de los padres fue mayor que el de las madres, en los tres períodos estudiados. Se observa además un incremento progresivo en el 
porcentaje de padres con educación superior y una disminución del porcentaje de los que solo tienen educación primaria. Entre los ingresantes el porcentaje de padres con educación superior ha sido siempre alto y se ha incrementado del $49 \%$ en el período 1962-64 al 72\% entre 1990-93.

CUADRO NN 8

POSTULANTES E INGRESANTES A LA FACULTAD DE MEDICINA POR GRADO DE INSTRUCCION DE LOS PADRES

\begin{tabular}{|c|c|c|c|c|c|c|c|c|}
\hline \multirow{2}{*}{ AÑO } & \multirow{2}{*}{$\begin{array}{l}\text { TOTAL } \\
\text { POST }\end{array}$} & \multicolumn{3}{|c|}{ \% POSTULANTES } & \multirow{2}{*}{\begin{tabular}{|l} 
TOTAL \\
INGRE
\end{tabular}} & \multicolumn{3}{|c|}{ \% INGRESANTES } \\
\hline & & Prim. & Sec. & Sup. & & Prim. & Sec. & Sup. \\
\hline \multicolumn{9}{|c|}{ GRADO DE INSTRUCCION DE LA MADRE } \\
\hline 1962 & 498 & 32 & 46 & 16 & 70 & 29 & 44 & 24 \\
\hline 64 & 492 & 20 & 52 & 16 & 60 & 20 & 48 & 17 \\
\hline Total 62-64 & 990 & 27 & 54 & 18 & 130 & 27 & 50 & 23 \\
\hline P.E.I. & & & & & & 11 & 11 & 16 \\
\hline 81 & 3190 & 27 & 43 & 30 & 69 & 16 & 36 & 48 \\
\hline 82 & 2877 & 25 & 44 & 31 & 71 & 14 & 56 & 30 \\
\hline 83 & 2781 & 23 & 43 & 34 & 73 & 11 & 36 & 53 \\
\hline Total $81-83$ & 8848 & 25 & 43 & 32 & 213 & 13 & 43 & 44 \\
\hline P.E.I. & & & & & & 1.3 & 2.4 & 3.3 \\
\hline 90 & 1970 & 14 & 40 & 46 & 59 & 3 & 36 & 61 \\
\hline 91 & 1794 & 16 & 37 & 47 & 60 & 7 & 35 & 58 \\
\hline 93 & 1074 & 9 & 50 & 41 & 142 & 5 & 46 & 49 \\
\hline Total 90-93 & 4838 & 14 & 41 & 45 & 261 & 5 & 41 & 54 \\
\hline P.E.I. & & & & & & 2 & 5.4 & 6.4 \\
\hline \multicolumn{9}{|c|}{ GRADO DE INSTRUCCION DEL PADRE } \\
\hline 1962 & 498 & 18 & 41 & 41 & 70 & 11 & 36 & 53 \\
\hline 64 & 492 & 16 & 44 & 40 & 60 & 17 & 38 & 45 \\
\hline Total 62-64 & 990 & 17 & 43 & 40 & 130 & 14 & 37 & 49 \\
\hline P.E.I. & & & & & & 10 & 10 & 15 \\
\hline 81 & 3190 & 18 & 31 & 51 & 69 & 12 & 16 & 72 \\
\hline 82 & 2877 & 16 & 31 & 53 & 71 & 11 & 32 & 57 \\
\hline 83 & 2781 & 14 & 31 & 55 & 73 & 6 & 28 & 66 \\
\hline Total $81-83$ & 8848 & 12 & 31 & 56 & 213 & 14 & 37 & 49 \\
\hline P.E.I. & & & & & & 2 & 2 & 3 \\
\hline 90 & 1970 & 10 & 26 & 64 & 59 & 2 & 18 & 80 \\
\hline 91 & 1794 & 10 & 26 & 64 & 60 & 2 & 20 & 78 \\
\hline 93 & 1074 & 7 & 34 & 58 & 142 & 2 & 32 & 66 \\
\hline Total $90-93$ & 4838 & 9 & 28 & 63 & 261 & 2 & 26 & 72 \\
\hline P.E.I. & & & & & & 1 & 5 & 6 \\
\hline
\end{tabular}

Fuente:Encuesta aplicada por la Comisión de Ingreso.

Al igual que en el caso de las madres, el P.E.I. fue siempre mayor para los postulantes cuyos padres tenían educación superior.

\section{DISCUSION}

La carrera de medicina sigue siendo considerada por la juventud peruana como "la mejor carrera" (9), ello ha contribuído a que el número de postulantes a medicina en el país se haya incrementado de 2,500 al año, en la década del 60, a 17,000 al año en la década del 90 (10).

En la UPCH, desde 1979 hasta 1994, más del 70\% de los postulantes solicitaron ser admitidos a medicina. Las variaciones en el número de postulantes observadas a lo largo de los años pueden haberse debido a factores propios de la Universidad o externos a ella. Para responder a esta interrogante comparamos las variaciones en el número de postulantes a las universidades del país y a la UPCH (Figura No8). En el período 196264 y entre 1979 y 1988 las variaciones en el número de postulantes a la UPCH fueron paralelas a las del conjunto de las universidades del país, sugiriendo que ellas dependieron de factores externos a la UPCH que afectaron por igual a todas las universidades. La caída en el número de postulantes en 1989, muy probablemente está 
relacionada con el drástico reajuste económico aplicado por el gobierno el año anterior, fenómeno que afectó en mayor grado a la UPCH y al resto de universidades, las causas de este fenómeno pueden ser tanto propias de la UPCH como externas a ella. Desafortunadamente no contamos con información sobre número de postulantes a las universidades del país a partir de 1992. El descenso en el número de postulantes a la UPCH que se observa a partir de 1992, coincide con la implantación del Ingreso Directo Exclusivo, modalidad de admisión en la cual los postulantes debieron matricularse en un ciclo semestral de estudios en el CEPU. El número de postulantes estuvo determinado por la demanda y la capacidad académica del CEPU, que se fijó en 900 vacantes por ciclo semestral.

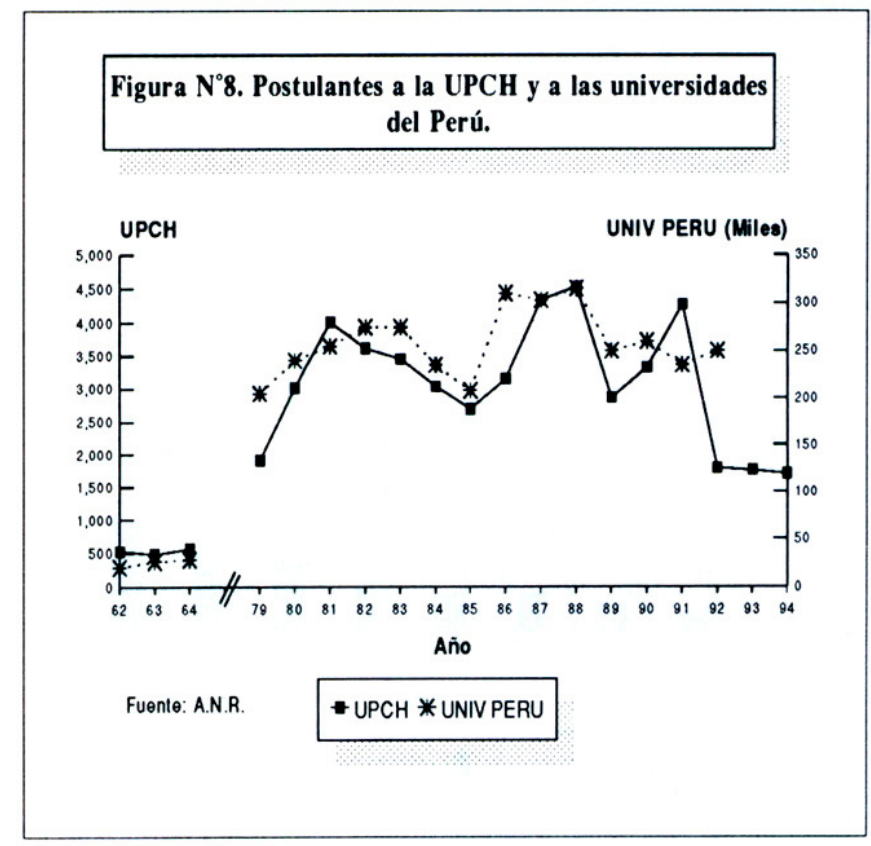

En el Perú la competencia por una vacante universitaria se ha mantenido en promedio en 3 postulantes por vacante entre 1960 y 1992 (4) y la competencia por una vacante de medicina ha sido superior al promedio, así entre 1980 y 1989 varió entre 13 y 29 postulantes/vacante (3)(10).

En la UPCH la competencia por una vacante de medicina, que en 1962 fue de 8 postulantes/vacante, se incrementó notablemente con el tiempo, así entre 1979 y 1991-I, en el Examen de Admisión la competencia estuvo entre 28 y 64 postulantes por vacante y en el Ingreso Directo, en el período 1987-1991-I, estuvo entre 23 y 34 postulantes por vacante de medicina.

Seleccionar con un adecuado grado de certeza a los mejores postulantes a través de un único examen, con un nivel tan alto de competencia, resulta tarea difícil. A partir de 1992, al implantarse la modalidad de Ingreso Directo Exclusivo, e incrementarse el número de vacantes, la competencia volvió a ser de 9 postulantes por ingresante. Lo anterior, unido a un proceso preparatorio uniforme para todos los postulantes a lo largo de un semestre y a la selección mediante la aplicación de cuatro exámenes parciales a lo 
largo del ciclo de estudios, creemos que ofreció a los postulantes más justas posibilidades de ingreso y a la UPCH la posibilidad de una mejor selección de alumnos de alto nivel académico. Esta hipótesis necesita ser analizada mediante estudios de seguimiento académico.

El número de ingresantes estuvo determinado por el número de vacante ofrecidas por la Facultad de Medicina. Para el primer proceso de selección, en 1962, la Facultad ofreció 70 vacantes, cifra que se mantuvo prácticamente sin variaciones a lo largo de 28 años, hasta 1989. Esta constancia en el número de vacantes de ingreso ha respondido a una política de la Facultad de Medicina, basada en la preocupación por preservar un nivel de excelencia en la formación profesional no superando su capacidad académica.

Paralelamente a los cambios descritos en cuanto a postulantes e ingresantes, la población del Perú se incrementó a más del doble, de 10 millones en 1961 a 22 millones en 1993. Por consiguiente el número de médicos requeridos también se incrementó, de 10,00 a 22,00, si tomamos en cuenta las recomendaciones de la OMS. Esta mayor demanda formal de médico ha sido cubierta en el país, ya que de 5,080 médicos con que contaba el país en 1964, se llegó a 25,000 en 1994 (11,12). Así mismo, el número de alumnos matriculados en facultades de medicina aumentó, de 2,500 al año entre 196064 al 12,5000 al año entre 1990-92 (10) y el número de médicos graduado por año se incrementó, de 406 en 1960 a 114 en 1994 (13). Este notable crecimiento en la formación de médicos se ha logrado en base a un incremento en el número de facultades de medicina de 3 a 1960, a 17 en 1994 (13), cuatro de ellas privadas (Figura No9). Es de suponer que muchas de estas facultades no cuentan con recursos materiales suficientes, y menos con personal docente idóneo para garantizar una formación profesional de calidad a los estudiantes. La profunda crisis económica experimentada por el país en los años que cubre el estudio y la falta de un organismo regulador y evaluador de los proyectos de creación de nuevas facultades son factores importantes que justificarían esta suposición. Como consecuencia de lo anterior se habría logrado satisfacer la demanda numérica sacrificando la calidad en la formación. Es necesario profundizar este análisis y preguntarse cuál debe ser la mejor respuesta de las universidades ante las crecientes necesidades de la sociedad en un período de grandes cambios.

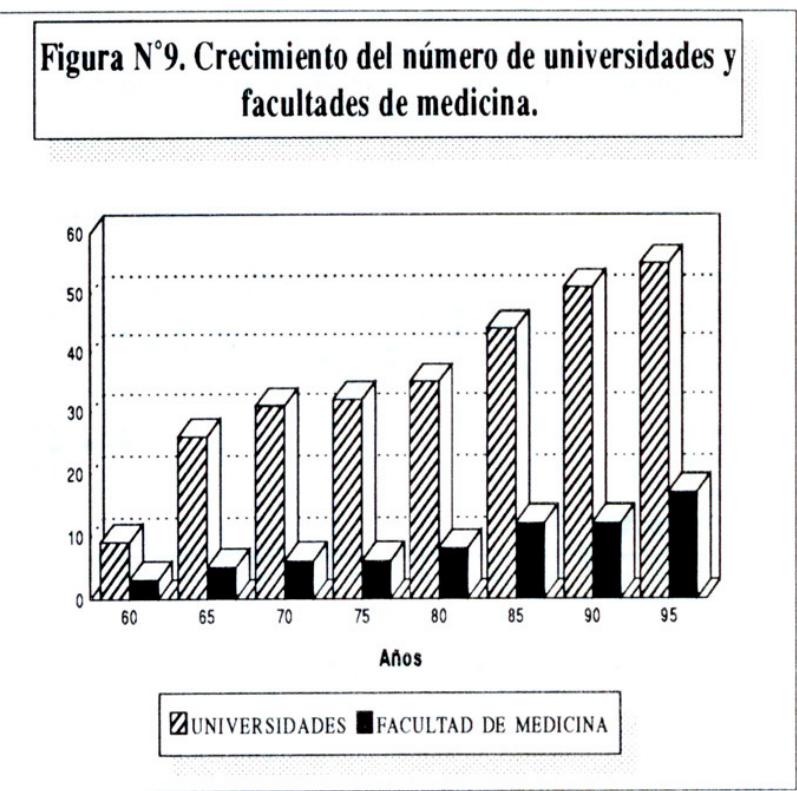


Otra de las variables estudiadas es la composición por sexo de los grupos de postulantes e ingresantes. En ambos grupos de postulantes e ingresantes. En ambos grupos se observa un incremento progresivo en el porcentaje de mujeres. A partir de 1990 entre los ingresantes a medicina en la UPCH, $33 \%$ fueron mujeres, cifra semejante a la del Censo de Alumnos 1993 que muestra que el 30\% de los alumnos matriculados en la Facultad de Medicina son mujeres (14). El progresivo incremento en la proporción de mujeres en la carrera de medicina es un fenómeno que se observa en otros países (15) y en general en todas las carreras universitarias, en el Perú (2) y en América Latina (6). Entre los médicos en ejercicio, encontramos que en 1994 el $20 \%$ eran mujeres, porcentaje que se irá incrementando, ya que a partir de 1989 las promociones de médicos que se inscriben en el Colegio Médico anualmente cuentan con $30 \%$ o más de mujeres (12).

En cuanto a edad, observamos que entre los ingresantes el porcentaje de jóvenes de 15 a 16 años ha disminuído notoriamente, de $32 \%$ en el período 1962-64 a 1\% en el período 1990-93, a pesar de que el porcentaje de postulantes de este grupo de edad que el porcentaje de postulantes de este grupo de edad se ha incrementado a lo largo de los tres períodos estudiados. Así mismo se ha incrementado el porcentaje de los ingresantes de 18 años o más. Lo anterior coincide con el hallazgo de que el mayor porcentaje de ingresantes terminó secundaria 2 ó más años antes de postular y también con que la mayor parte de los ingresantes postuló dos o más veces a la UPCH antes de ingresar.

El tiempo transcurrido entre la terminación de la secundaria y el ingreso a la carrera de medicina probablemente es empleado por la mayor parte de los postulantes para prepararse en un centro preuniversitario. Un indicador de la importancia que la preparación pre-universitaria ha tomado como completo de la educación escolar lo encontramos en un estudio anterior (8) que mostró que entre 1981 y 1983 , el 90\% de los postulantes y el 95\% de los ingresantes a la UPCH se preparó en una academia preuniversitaria. Las principales causas de éste fenómeno creemos que son la elevada competencia por una vacante de ingreso y las deficiencias de la educación escolar.

Hemos analizado también la influencia del tipo de colegio de procedencia sobre la probabilidad de ingreso a medicina en la UPCH, encontrando que esta disminuyó marcadamente para los egresados de colegios estatales a lo largo de los años, mientras que el porcentaje de postulantes de este tipo de colegios disminuyó sólo moderadamente. Este fenómeno parece indicar un mayor deterioro de la educación escolar estatal que de la particular.

La disminución de postulantes de colegios estatales observada entre 1992 y 1993 puede estar relacionada con la implantación de la modalidad de Ingreso Directo Exclusivo, que supuso un ciclo semestral de estudios en el CEPU, hecho que pudo hacer desalentado a los postulantes con menor nivel académico y con menores recursos económicos, a pesar del elevado número de becas de estudios que se ofrecieron. 
Desde 1962 a la fecha, el 70\% de los postulantes y el 80\% de los ingresantes provienen de colegios ubicados en Lima y Callao. Es decir que la zona de influencia de la UPCH en el país no ha variado.

También analizamos el grado de instrucción de los padres, común indicador de la influencia del hogar sobre las probabilidades de ingreso, encontrando que el mayor Porcentaje de Éxito al Ingreso lo tiene el postulante cuyo padre y/o madre tiene educación superior.

En este estudio presentamos información sobre los cambios ocurridos en el perfil de los postulantes e ingresantes a medicina en la UPCH y su relación con los cambios de la sociedad y del sistema educativo. La naturaleza de la información con que contamos nos permite solamente sugerir algunas explicaciones a los cambios observados. Consideramos que las tendencias de los cambios en las variables estudiadas son válidas $\mathrm{y}$ deben tenerse en cuenta al analizar la problemática del ingreso a la carrera de medicina.

\section{Correspondencia:}

Dra. Graciela Risco de Domínguez

Universidad Peruana Cayetano Heredia

Av. Honorio Delgado 430,

San Martín de Porres

Lima 31, Perú.

\section{REFERENCIAS BIBLIOGRÁFICAS}

1.Querol M, Castro de la Mata R, Ishiyama R, et al. Métodos de selección y rendimiento académico. Informe presentado por la Comisión Técnica de Selección de la UPCH. Lima, Perú: Universidad Peruana Cayetano Heredia, 1965.

2.Instituto Nacional de Estadística e Informática (INEI). Compendio de Estadísticas Sociales 1993-1994. Lima, Perú, Setiembre 1994.

3.Asamblea Nacional de Rectores (ANR). Perú, Estadísticas Universitarias 1960-86. Boletín Estadístico Nº10. Lima, Perú: Asamblea Nacional de Rectores. 1988.

4.Mc Lauchlan de Arregui P. La Situación de las Universidades Peruanas. En: GuerraGarcia R, Editor Diálogo sobre la Universidad Peruana, UPCH y ANR, Lima, Perú, 1994: 23-50.

5.GRADE, Educación Superior en el Perú: Datos para el Análisis. Documento de Trabajo Nº. Lima, Perú, 1990.

6.Brunner J. La gran transformación en Educación superior en América Latina: Cambios y desafíos. Santiago de Chile; Fondo de Cultura Económica.1990: 71-132.

7.Universidad Peruana Cayetano Heredia. Informes sobre la Encuesta Demográfica y Socioeconómica aplicada a los postulantes al concurso de Ingreso a la UPCH, 1981, 1982 y 1983. Elaborado por las respectivas Comisiones de Ingreso.

8.Risco de Domínguez G. Análisis comparativo de la encuesta Socioeconómica Aplicada a los Postulantes a Ingreso a la UPCH a lo largo de tres años (1981-1983). Informe elaborado a solicitud de la Comisión de Ingreso. Lima, Perú, 1983.

9.APOYO S.A. Perfil del Mercado Educativo 1994. Lima Perú, 1994.

10.GRADE. Educación Superior en el Perú: Datos para el Análisis II. Lima, Perú, 1994. 
11.Lip C, Lazo O, Brito P. El Trabajo Médico en el Perú. Lima Perú: OPS - UPCH. 1990.

12.Colegio Médico del Perú, Consejo Nacional. Informe Estadístico preparado por la División de Contabilidad. Lima: Colegio Médico del Perú. Enero 1995.

13.Asamblea Nacional de Rectores (ANR). Universidades del Perú. Facultades y Carreras profesionales, Grados y Títulos que se Otorgan, 1992. Dirección de Estadística e Informática. Organo de Difusión $N^{\circ}$. Lima, Perú: Asamblea Nacional de Rectores 1992.

14.Universidad Peruana Cayetano Heredia. Censo de Alumnos de Pre-Grado 1993. Lima, Perú: Universidad Peruana Cayetano Heredia. Marzo 1994.

15.The American College of Physicians. Promotion and Tenure of Women and Minorities on Medical School Faculties. Ann Int Med 1991; 114: 63-68. 